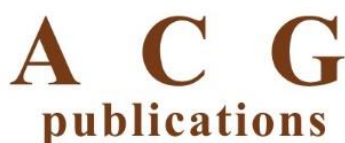

Org. Commun. 14:4 (2021) 346-354 organic communications

\title{
Effect of reactor configurations on the Suzuki cross-coupling reaction using a carrageenan-based $\mathrm{RhCl}_{3}$ catalyst
}

\author{
Oshrat Levy-Ontman $\oplus^{*}$, Eduard Arbit $\odot$, Sivan Leviev ${ }^{\odot}$ and \\ Adi Wolfson
}
${ }^{1}$ Department of Chemical Engineering, Shamoon College of Engineering, Basel/Bialik Sts., Beer-Sheva 8410001, Israel

(Received August 28, 2021; Revised September 22, 2021; Accepted September 29, 2021)

\begin{abstract}
RhCl}_{3}$ was heterogenized into an iota-carrageenan polysaccharide support, and the effect of various reactor configurations on catalyst performance in a Suzuki cross-coupling reaction was studied. It was found that performing the reaction in a high-volume, well-agitated, mechanically mixed high volume reactor, or circulating the reaction mixture through the catalyst, which was placed in a tabular reactor, yielded higher product yields in comparison to magnetically stirring or shaking. In addition, the catalyst was also successfully recycled in all the systems. Moreover, the reaction solution in each reactor configuration did not contain any traces of rhodium, while elemental composition of the iota-based rhodium catalyst, $i$ - $\mathrm{RhCl}_{3}$, following the first and the second cycle in the glass tubular reactor was similar to the fresh $\mathrm{i}-\mathrm{RhCl}_{3}$ catalyst, demonstrating the high xerogel stability.
\end{abstract}

Keywords: Heterogeneous catalysis; polysaccharides; rhodium; Suzuki cross-coupling. @2021 ACG Publication. All right reserved.

\section{Introduction}

Transition metal complexes (TMCs) are very active, selective and versatile catalysts for organic syntheses. ${ }^{1,2}$ Yet their separation from the reaction mixture and recycling is usually difficult to accomplish. Thus, despite their evident advantages, TMCs have found limited use to date in large-scale industrial applications.

TMC heterogenization is a well-known and extensively studied method, yielding a heterogeneous analog to the homogenous catalyst. ${ }^{3-5}$ An optimal heterogenized catalyst ought to have all the advantages of homogeneous catalysts, including high performance, simple separation and reusability. A variety of inorganics and organics supports have been employed for TMC heterogenization. These include renewable and biodegradable polysaccharides produced by various living organisms, which have found many applications nowadays. ${ }^{6-10}$

Among the variety of heterogeneous systems, palladium-based polysaccharide heterogeneous catalysts have been widely investigated, ${ }^{11-14}$ and most of these new palladium catalysts have been tested in the Suzuki cross-coupling of halobenzens and organoborons. ${ }^{15-20}$ Yet, over the years, other metals, such as rhodium, ${ }^{21,22}$ platinum, ${ }^{23}$ nickel, ${ }^{24,25}$ and copper, ${ }^{26}$ have also been tested in a Suzuki crosscoupling, and their applications within heterogeneous catalytic systems have thereby also been investigated.

We recently synthesized several heterogeneous catalysts by immobilizing palladium and rhodium salts and complexes in polysaccharide matrixes, yielding 'sponge-like' xerogels. ${ }^{27-31}$ These

\footnotetext{
* Corresponding author: E-Mail: oshrale@ @sce.ac.il, Phone: + 972 86475207; Fax: + 97286475636. 
catalysts were successfully used, separated and recycled in the Suzuki cross-coupling reaction. Furthermore, rhodium chloride was immobilized on iota-Carrageenan (i) polysaccharide without the addition of a ligand i.e., $i$ - $\mathrm{RhCl}_{3} \cdot{ }^{31} \mathrm{In}$ addition, it was demonstrated by XPS analysis that the rhodium oxidation state, $\mathrm{Rh}^{+3}$, was not changed during the immobilization or the reaction, and that the atomic ratio of $\mathrm{Rh}: \mathrm{Cl}$ in the heterogeneous catalyst was around 3:1. Furthermore, the rhodium was immobilized to the $i$ via the sulfate groups on its backbone without any ligand. Thus it offers very simple, cheap and more environmentally friendly system, in comparison to palladium.

We report in this paper on the use of $\mathrm{i}-\mathrm{RhCl}_{3}$ as a heterogeneous catalyst in Suzuki crosscoupling of iodobenzene and phenylboronic acid, using various high-volume laboratory reactor configurations.

\section{Experimental}

\subsection{Reagents and Polysaccharide Supports}

All chemicals (analytical grades) and $i$ polysaccharide were purchased from Sigma-Aldrich Israel.

\subsection{Reagents and Polysaccharide Supports}

The heterogeneous catalyst was prepared as described before. ${ }^{30}$ Briefly, $1 \mathrm{~g}$ of $i$ was dissolved in $100 \mathrm{~mL}$ double-distilled water until a homogeneous solution was obtained, and was added to a vial with $10 \mu \mathrm{mol}$ or $20 \mu \mathrm{mol} \mathrm{RhCl}_{3}$ in $3 \mathrm{~mL} \mathrm{DDW}$ and mixed by vortexing for homogenization. It was then poured into a $15 \mathrm{~mL}$ polypropylene tube, sealed, mixed, and deep-freezed at $-20{ }^{\circ} \mathrm{C}$ for $24 \mathrm{~h}$, until all the liquid was frozen. Finally, the seal was removed and the tube was covered with a paraffin sheet pierced by a disposable toothpick and lyophilized for $48 \mathrm{~h}$, yielding a 'sponge-like' xerogel that was cut into $\sim 1 \mathrm{~cm} \times 1 \mathrm{~cm}$ pieces and added to the reaction mixture.

\subsection{Reaction Procedure}

In a typical procedure, $20 \mu \mathrm{mol}$ of $\mathrm{RhCl}_{3}$ (homogenous or heterogeneous in the form of 1 xerogel with $20 \mu \mathrm{mol}$ catalyst, i.e., $20 \mu \mathrm{mol} i$-RhCl 3 or 2 xerogels with $10 \mu \mathrm{mol}$ catalyst, i.e., $2 \times 10 \mu \mathrm{mol} i$ $\mathrm{RhCl}_{3}$ ) were added to a glass vessel, e.g., a $10-250 \mathrm{~mL}$ beaker or $250 \mathrm{~mL}$ Erlenmeyer, or a $1 \mathrm{~L} \mathrm{Buchi}$ glass reactor, with 5-200 mL ethanol together with $0.5 \mathrm{~g}$ iodobenzene, $0.45 \mathrm{~g}$ phenylboronic acid and $0.34 \mathrm{~g} \mathrm{Na}_{2} \mathrm{CO}_{3}$. In the beaker system, an up to $200 \mathrm{~mL}$ reaction mixture was placed in a preheated oil bath at $50{ }^{\circ} \mathrm{C}$ and magnetically stirred at $100 \mathrm{rpm}$ for $24 \mathrm{~h}$. Alternatively, a $250 \mathrm{~mL}$ Erlenmeyer with a $200 \mathrm{~mL}$ reaction mixture was placed in a Julabo SW22 shaking water bath, and shaken at $100 \mathrm{rpm}$ and $50{ }^{\circ} \mathrm{C}$ for $24 \mathrm{~h}$. In the third system, a $1 \mathrm{~L} \mathrm{Buchi} \mathrm{glass} \mathrm{reactor} \mathrm{(diameter}=164 \mathrm{~mm}$ ) with a propeller stirrer (diameter $=60 \mathrm{~mm}$ ) was filled with a $200 \mathrm{~mL}$ reaction mixture and kept at $100 \mathrm{rpm}$ and $50{ }^{\circ} \mathrm{C}$ for $24 \mathrm{~h}$.

The organic phase was analyzed during the reaction to determine the reaction product yield by gas chromatography (GC) by using a HP-5 column. The average sum-square peak ratios were calculated according to a calibrated reactant-product curve. At the end of the reaction, the reaction mixture was cooled to room temperature.

Another system was built of a $2.5 \mathrm{~mm} \times 20 \mathrm{~mm}$ tabular glass reactor connected by Teflon pipes to a $250 \mathrm{~mL}$ glass vessel with two entrances and a Cole Parmer peristaltic pump. The xerogel catalysts (20 $\mu \mathrm{mol} i-\mathrm{RhCl}_{3}$ or $2 \times 10 \mu \mathrm{mol} i-\mathrm{RhCl}_{3}$ or $2 \times 20 \mu \mathrm{mol}$ of $i-\mathrm{RhCl}_{3}$ ), was situated in the middle of a pipe and trapped by glass wool from both sides. The reactants, $0.5 \mathrm{~g}$ iodobenzene, $0.45 \mathrm{~g}$ phenylboronic acid and $0.34 \mathrm{~g} \mathrm{Na}_{2} \mathrm{CO}_{3}$, were added together with $200 \mathrm{ml}$ ethanol to the vessel, which was placed on a heating and mixing plate. Finally, the glass vessel and the glass reactor were heated to $50{ }^{\circ} \mathrm{C}$ in Julabo oil baths. The reaction mixture was circulated through the catalyst by the pump and samples were taken from the vessel and analyzed by GC with a HP-5 column during running the reaction.

\subsection{Leaching Tests and Catalyst Recycling}

Leaching of the catalysts was tested by either performing a second reaction after the catalyst was removed from the primary reaction mixture, while adding corresponding amounts of fresh 
substrates and sodium carbonate and mixing for $24 \mathrm{~h}$ under similar conditions, or using ICP-OES (Arcos, Spectro) analysis for rhodium leftovers in the reaction medium after the first cycle.

Catalyst recycling was performed by filtrating the catalyst after the first cycle and adding the recovered catalyst to a fresh solution with substrates and base. In the tabular reactor, the catalyst recycling was performed by replacing the reaction mixture with a fresh one (containing the reactants and a base).

\subsection{Analyses}

Surface Analysis by X-ray Photoelectron Spectroscopy (XPS). XPS measurements were performed by using an X-ray photoelectron spectrometer ESCALAB 250 ultrahigh vacuum $\left(1 \times 10^{-9}\right.$ bar) apparatus with an AlK $\alpha$ X-ray source and a monochromator. The spot size of the X-ray was 500 $\mu \mathrm{m}$, and survey spectra were recorded with a pass energy (PE) of $150 \mathrm{eV}$ while high energy resolution spectra were recorded with a pass energy (PE) of $20 \mathrm{eV}$. To correct for charging effects, all spectra were calibrated relative to a carbon C 1 s peak positioned at $284.8 \mathrm{eV}$. The XPS results were processed with AVANTGE software.

Scanning Electron Microscope (SEM analysis). Scanning electronic microscopy (SEM) was performed with a Phenom XL Scanning Electron Microscope and a backscatter electron detector equipped with an energy-dispersive X-ray spectroscopy detector (Thermo Fisher). Acceleration voltage was $15 \mathrm{kV}$ and sample pressure $0.1 \mathrm{~Pa}$.

Transmission electron microscope (TEM) Analysis. High-resolution transmission electron microscopic (HRTEM) micrographs of $\mathrm{i}-\mathrm{RhCl}_{3}$ system were obtained using an EFI Talos F200C transmission electron microscope (TEM) operated at $200 \mathrm{kV}$ at room temperature. The samples were prepared by deposition of a drop of ethanol suspension of the crushed sponge catalyst on a carboncoated $\mathrm{Cu}$ grid.

\section{Results and Discussion}

\subsection{Chemistry}

The investigation began by performing a homogeneous reaction in ethanol, using $\mathrm{RhCl}_{3}$ as the catalyst (Table 1). As biphenyl can be also synthesized via homo-coupling of phenylboronic acid or iodobenzene, (i.e. Ullmann coupling), reference reactions with or without the addition of $\mathrm{RhCl}_{3}$, using either phenylboronic acid or iodobenzene, were first tested and did not yield any biphenyl. The reactions were first performed under magnetic stirring (MS) in different volumes, from $50 \mathrm{~mL}$ to $200 \mathrm{~mL}$ (Table 1, entries 1-3), with a constant substrate to catalyst ratio, but with different substrates and catalyst concentrations. As illustrated in Table 1, it was found that changing the reaction volume only slightly changed the product yields.

Table 1. Comparison of homogeneous and heterogeneous reactions with $i-\mathrm{RhCl}_{3}$ in different systems ${ }^{1}$

\begin{tabular}{ccccccc}
\hline Entry & Mode & System & $\begin{array}{c}\text { Volume } \\
(\mathbf{m L})\end{array}$ & $\begin{array}{c}\text { Product } \\
\text { yield (\%) }\end{array}$ & $\begin{array}{c}\text { Product } \\
\text { yield }(\%)^{\mathbf{2}}\end{array}$ & $\begin{array}{c}\text { Product } \\
\text { yield (\%) }\end{array}$ \\
\hline 1 & Homogeneous & MS & 50 & 65 & - & - \\
2 & Homogeneous & MS & 100 & 64 & - & - \\
3 & Homogeneous & MS & 200 & 62 & - & - \\
4 & Heterogeneous & MS & 200 & 18 & 19 & 19 \\
5 & Homogeneous & SH & 200 & 63 & - & - \\
6 & Heterogeneous & SH & 200 & 22 & 21 & - \\
7 & Heterogeneous & SH & 200 & 20 & - & 40 \\
8 & Heterogeneous & MSR & 200 & 48 & 42 & 4 \\
\hline
\end{tabular}

${ }^{1}$ Reaction conditions, $0.5 \mathrm{~g}$ iodobenzene, $0.45 \mathrm{~g}$ phenylboronic acid, $0.34 \mathrm{~g} \mathrm{Na}_{2} \mathrm{CO}_{3}, 2 * 10 \mu \mathrm{mol} i$ - $\mathrm{RhCl}_{3}$, ethanol,

$50{ }^{\circ} \mathrm{C}, 24 \mathrm{~h}$.

${ }^{2} 1^{\text {st }}$ reuse.

${ }^{3} 2^{\text {nd }}$ reuse.

${ }^{4} 20 \mu \mathrm{mol} i-\mathrm{RhCl}_{3}$. 
As expected, ${ }^{31}$ performing the heterogeneous reaction with $i-\mathrm{RhCl}_{3}$ under MS (Table 1 , entry 4 ) yielded lower product yields compared to the homogeneous reaction (Table 1, entry 3), 18\% and 62\% after $24 \mathrm{~h}$, correspondingly, probably due to mass transfer limitation of the substrates and the product through the support. However, it is worth mentioning that the catalyst was seen to float on top of the solvent, regardless the shape of the vessel, e.g., round glass or Erlenmeyer. Thus, in order to increase the dispersion of the sponge pieces in the solvent, the reaction was also performed in a high volume under shaking conditions ( $\mathrm{SH})$. As it turns out, the heterogeneous catalyst was dispersed more effectively in the Erlenmeyer that was shaken, yet the product yield of the heterogeneous system was only slightly higher, reaching $22 \%$ (Table 1, entry 6). Moreover, it was still much lower than that of the corresponding homogeneous system (Table 1, entry 5), which yielded a product yield similar to that of the reaction under magnetic stirring (Table 1, entry 3 ). Furthermore, using only a single sponge with 20 $\mu \mathrm{mol}$ catalysts instead of two sponges with $10 \mu \mathrm{mol}$ catalyst resulted in only a slightly lower product yield (Table 1, entry 7). This might be attributed to the lower dispersion of the catalyst in the support.

Finally, the Suzuki cross-coupling of iodobenzene and phenylboronic acid was also performed in a mechanically stirred reactor (MSR), equipped with a jacket, where agitating and heat transfer are expected to be much more efficient (Table 1, entry 8). The first step was to find the optimal stirring speed (rpm) that could be applied without crushing the xerogel. It was found that no significant difference was observed between $50 \mathrm{rpm}$ and $100 \mathrm{rpm}$, whereas a further increase in the stirring speed to $200 \mathrm{rpm}$ led to a vortex and crushed the xerogel so it could not be recycled. Thus, $100 \mathrm{rpm}$ was chosen as the stirring speed. This time, not only was the dispersion of the xerogel in the reaction mixture much greater, the reaction product yield after $24 \mathrm{~h}$ was also much higher: $48 \%$, a two-fold increase over magnetic string (Table 1, entry 4) or shaking (Table 1, entry 6). This stresses the importance of efficient momentum, heat and mass transfer and the huge effect that reactor configuration has on catalytic performance.

The new heterogeneous catalyst was also separated and reused in the three different systems. It was demonstrated that the product yields at the end of the $2^{\text {nd }}$ cycle in each of the configurations (after $48 \mathrm{~h}$ ) were almost the same as in the ${ }^{\text {st }}$ cycle, while the product yields of the $3^{\text {rd }}$ cycle (after $72 \mathrm{~h}$ ) were slightly lower (Table 1). In all the three systems, the recycling was performed after filtration of the catalyst from the reaction mixture, followed by an addition of the heterogeneous catalyst to a fresh mixture of substrates in ethanol; thus the low decrease in product yield might be attributed to some loss of catalyst during filtration. Yet the recycling results demonstrate that the catalyst did not lose its activity and was well heterogenized.

As the configuration of the reactor has a profound effect on catalyst performance, and continuous reactors are an important class of reactors, we also investigated the Suzuki cross-coupling with the $i-\mathrm{RhCl}_{3}$ catalyst in a glass tabular reactor. Here, the reaction mixture was circulated through the catalysts continuously. Figure 1 illustrates the product yield change with the reaction time, using two sponges of iota with $10 \mu \mathrm{mol} \mathrm{RhCl} 3$, one sponge with $20 \mu \mathrm{mol}$ of $\mathrm{RhCl}_{3}$, and 2 sponges with $20 \mu \mathrm{mol}$ of $\mathrm{RhCl}_{3}$. It can be seen that after $24 \mathrm{~h}$ the product yields were $41 \%, 43 \%$, and $62 \%$, correspondingly, much higher than that of the corresponding stirred and shaken systems (Table 1). In addition, as can be seen in Figure 1, again using two sponges with $10 \mu \mathrm{mol}$ catalyst each, product yields were almost the same as one sponge with $20 \mu \mathrm{mol}$ of catalyst. Furthermore, employing two sponges with $20 \mu \mathrm{mol}$ of $\mathrm{RhCl}_{3}$ each yielded higher product yield of $62 \%$ after $24 \mathrm{~h}$, yet the product yield was not doubled. Finally, the reaction mixtures of the various cycles in all the configurations were run for an additional $24 \mathrm{~h}$ under similar reaction conditions after removal of the catalyst by filtration. In all the heterogeneous reactions the product yields after removal of the catalyst and mixing for an additional $24 \mathrm{~h}$ were unchanged. Furthermore, ICP-OES detection of the reaction mixtures after $24 \mathrm{~h}$ reaction and removal of the heterogonous catalysts did not show any rhodium leftovers, as rhodium content was below the detection limit $(<1 \mathrm{ppm})$, which indicates that the catalyst was indeed heterogeneous. Therefore, the recycling results prove that changing the reactor configuration has no effect on the activity of the catalyst after running the reaction in the form of a polysaccharide-based xerogel, thus demonstrating its superior stability. 


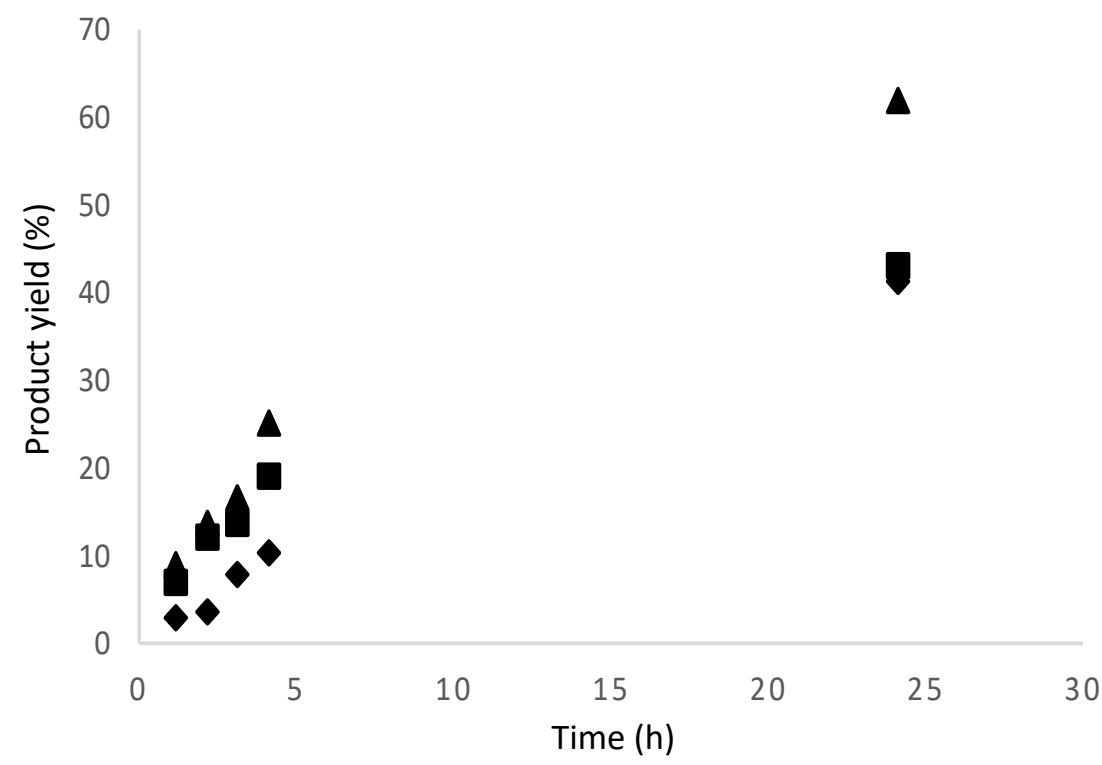

Figure 1. Reaction product yields in a tubular system: (४) $2 \times 10 \mu \mathrm{mol} i$-RhCl ${ }_{3}$, (匹) $20 \mu \mathrm{mol} i$ - $\mathrm{RhCl}_{3}$, ( $\Delta$ ) $2 \times 20 \mu \mathrm{mol} i-\mathrm{RhCl}_{3}$. Reaction conditions, $0.5 \mathrm{~g}$ iodobenzene, $0.45 \mathrm{~g}$ phenylboronic acid, $0.34 \mathrm{~g} \mathrm{Na}_{2} \mathrm{CO}_{3}, 2 \times 10 \mu \mathrm{mol} i-\mathrm{RhCl}_{3}, 200 \mathrm{~mL}$ ethanol, $50{ }^{\circ} \mathrm{C}$

In order to gain more insight into the stability of the heterogeneous catalyst and to evaluate its effectiveness as a reusable catalyst, it was also very important to confirm that the rhodium was embedded in the polysaccharide matrix. Indeed, an XPS survey spectrum of $i$ - $\mathrm{RhCl}_{3}$ confirms that all the elements used to form the 'sponge-like' xerogel catalyst are present (Figure 2). The elemental ratios between the elements was also similar to previously published data ${ }^{31}$. As expected, focusing on the XPS spectrum of $i-\mathrm{RhCl}_{3}$ in the $\mathrm{Rh} 3 \mathrm{~d}$ region yielded a typical doublet that indicated that the rhodium exists in the oxidation state of +3 (i.e. $\mathrm{Rh}^{3+}$ ). The atomic percentage of the rhodium measured within the sample was $0.6 \%$, which was in line with our previous publications ${ }^{31}$.

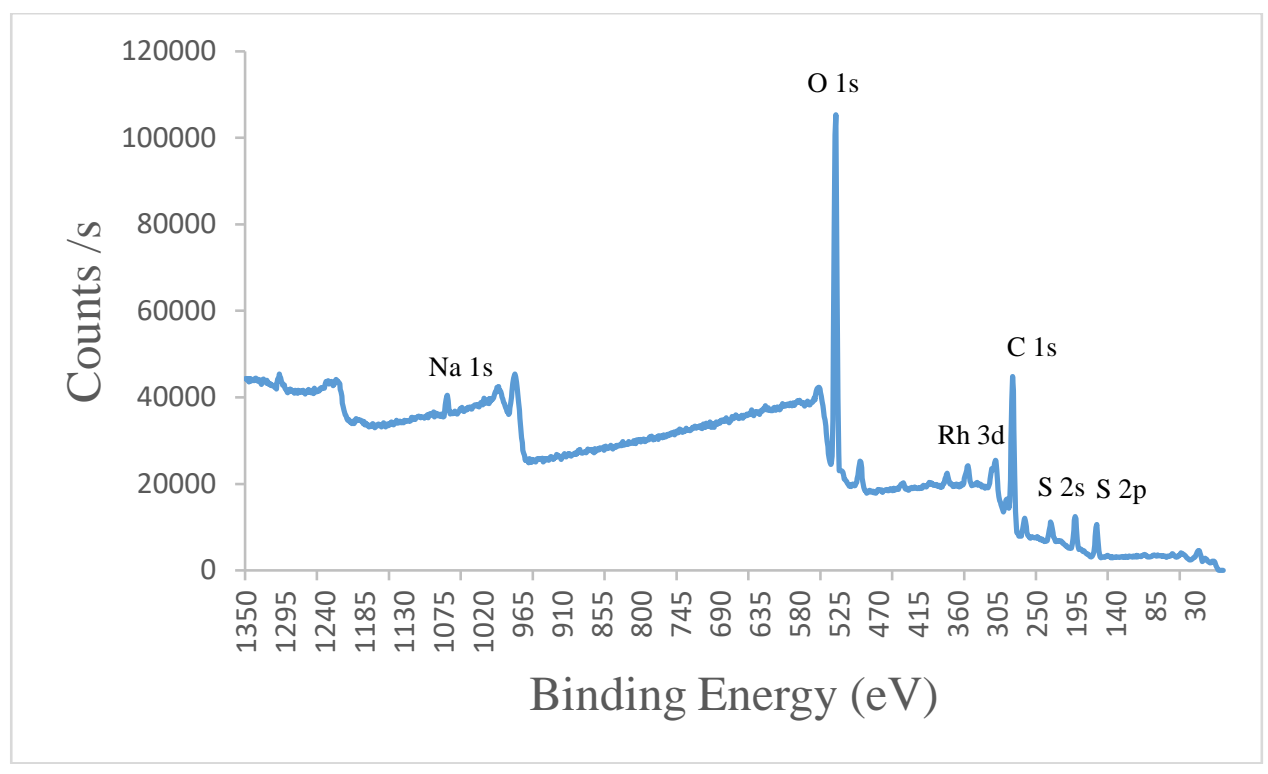

Figure 2. XPS survey spectrum for $i-\mathrm{RhCl}_{3}$ before reaction

The next step was to study the changes in the heterogeneous catalyst after the $1^{\text {st }}$ and the $2^{\text {nd }}$ cycles in the tubular system. Accordingly, the morphology and the elemental composition of $i-\mathrm{RhCl}_{3}$ before the reaction (i.e. fresh $i-\mathrm{RhCl}_{3}$ ) and following the 1 st cycle (after $24 \mathrm{~h}$ of reaction conditions) and the $2^{\text {nd }}$ cycle (after $48 \mathrm{~h}$ ), was detected using SEM-EDS. At first, cross-sectional pieces of the different 
samples were prepared by cutting the sponge into thin slices. All the slices were characterized by a similar structure, composed of an ordered porous surface (Figure 3). Notably, the exposure of the catalyst to the reaction conditions only slightly affected sponge morphology, as was demonstrated by an increase of void space in the polysaccharide matrix when the catalyst was exposed to reaction conditions for a longer time.
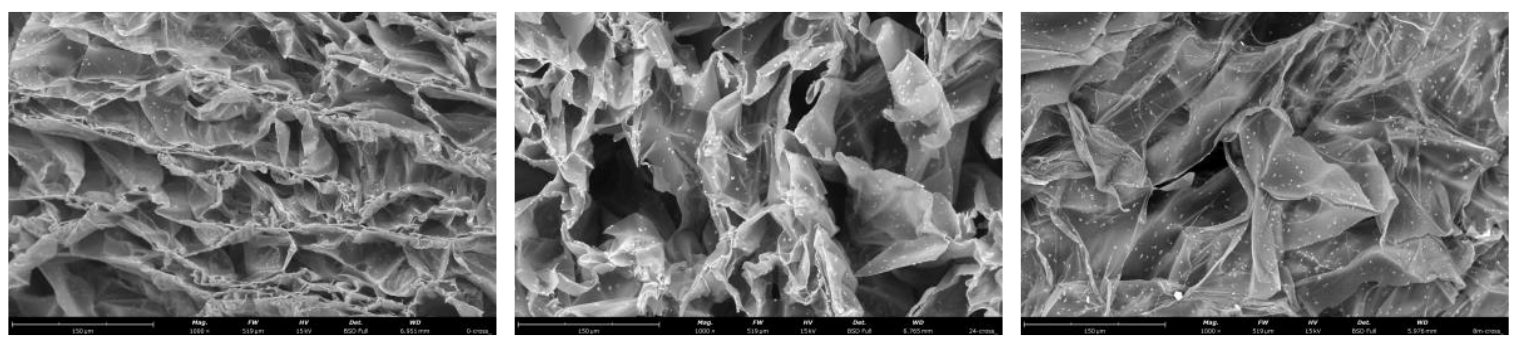

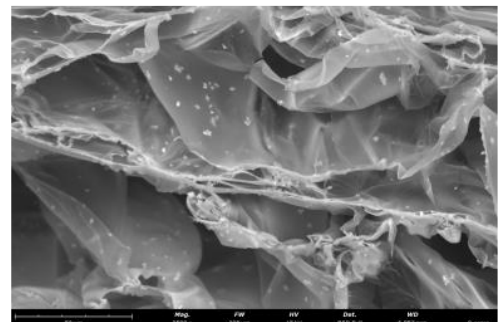

(a)

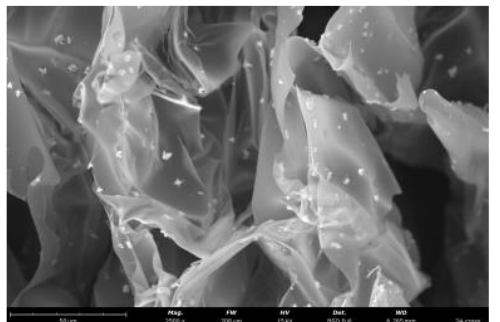

(c)

(b)

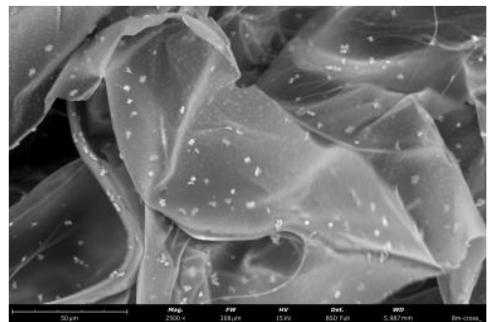

Figure 3. SEM analysis of cross-sectional pieces of $i$ - $\mathrm{RhCl}_{3}$ catalyst (a) before reaction; (b) after the 1 st cycle (after $24 \mathrm{~h}$ ) and (c) after the 2 nd cycle (after $48 \mathrm{~h}$ ) in the glass tubular reactor

The composition of the rhodium in the xerogel after the $1^{\text {st }}$ and the $2^{\text {nd }}$ cycles was similar to the fresh $i-\mathrm{RhCl}_{3}$ catalyst xerogel $(\sim 3.00 \% \mathrm{wt})$ (Table 2$)$. A slight change in $\mathrm{S}: \mathrm{Rh}$ ratio was observed following the $2^{\text {nd }}$ cycle in comparison to the fresh $i-\mathrm{RhCl}_{3}$ or to the catalyst after the $1^{\text {st }}$ cycle. This phenomenon may be attributed to sintering of the rhodium, which could occur due to continuous heating. These results demonstrate that the xerogel did not change its basic compositional structure and exhibited a stabilized structure during the reaction.

Table 2. Elemental composition of $i-\mathrm{RhCl}_{3}$ before reaction and following the $1^{\text {st }}$ and $2^{\text {nd }}$ cycles

\begin{tabular}{|c|c|c|c|c|c|c|}
\hline \multirow[b]{2}{*}{$\begin{array}{c}\text { Element } \\
\text { Symbol }\end{array}$} & \multicolumn{2}{|c|}{ Before Reaction } & \multicolumn{2}{|c|}{ After the $1^{\text {st }}$ cycle } & \multicolumn{2}{|c|}{ After the $2^{\text {nd }}$ cycle } \\
\hline & $\begin{array}{c}\text { Atomic } \\
\%\end{array}$ & $\begin{array}{c}\text { Weight } \\
\%\end{array}$ & $\begin{array}{c}\text { Atomic } \\
\%\end{array}$ & $\begin{array}{c}\text { Weight } \\
\%\end{array}$ & $\begin{array}{c}\text { Atomic } \\
\%\end{array}$ & $\begin{array}{c}\text { Weight } \\
\%\end{array}$ \\
\hline $\mathrm{C}$ & 34.7 & 21.7 & 32.6 & 20.3 & 35.1 & 23.2 \\
\hline $\mathrm{O}$ & 42.6 & 35.5 & 45.2 & 37.5 & 47.6 & 41.9 \\
\hline $\mathrm{Na}$ & 1.42 & 1.70 & 1.00 & 1.20 & 1.13 & 1.40 \\
\hline $\mathrm{S}$ & 9.53 & 15.9 & 9.50 & 15.8 & 7.19 & 12.7 \\
\hline $\mathrm{Cl}$ & 2.06 & 3.80 & 2.77 & 5.10 & 2.51 & 4.91 \\
\hline $\mathrm{K}$ & 3.58 & 7.03 & 5.56 & 11.3 & 3.76 & 8.10 \\
\hline $\mathrm{Ca}$ & 5.61 & 11.7 & 2.83 & 5.90 & 2.18 & 4.79 \\
\hline $\mathrm{Rh}$ & 0.50 & 2.67 & 0.54 & 2.90 & 0.53 & 3.00 \\
\hline
\end{tabular}

The fresh catalyst and the catalyst that were obtained after $24 \mathrm{~h}$ of reaction time in the glass tubular reactor were also analyzed by TEM. The TEM micrograph of $i-\mathrm{RhCl}_{3}$ before the reaction was characterized by an unordered micro-structure, and no rhodium nanoparticles were detected (Figure 4a), while the TEM image of $i$ - $\mathrm{RhCl}_{3}$ after the reaction also yielded a similar structure (Figure $4 \mathrm{~b}$ ), yet with small particles that might be attributed to a change in the polysaccharide support. In addition, the shape of the sponge after the reaction was more symmetric and a bit lighter with spots in different colors, which indicate that the texture of the sponge had changed. These observations suggest that the sponge kept its form under these reaction conditions, indicating that the $i-\mathrm{RhCl}_{3}$ is stable and it is suitable to be used as heterogeneous catalyst. 


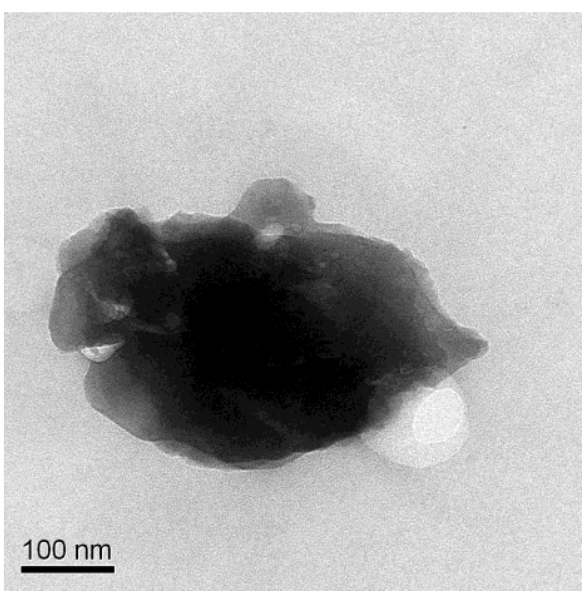

(a)

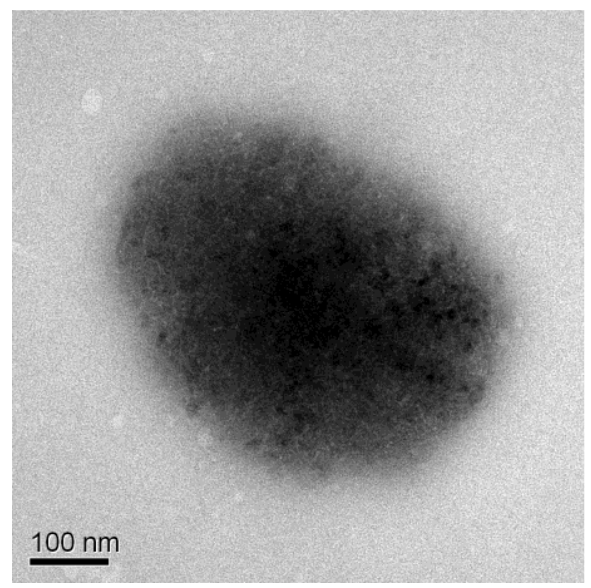

(b)

Figure 4. TEM micrographs of (a) fresh $i-\mathrm{RhCl}_{3}$, (b) $i-\mathrm{RhCl}_{3}$ after reaction

The $i-\mathrm{RhCl}_{3}$ catalyst used in this study was chosen as a representative catalyst to compare the different configurations. To improve the catalytic activity, other metals or TMCs, which have higher activity in a Suzuki cross coupling, can be used. In addition, improving the preparation of the polysaccharide support and increasing the porosity and the surface area, for instance by preparing an aerogel, might increase the product yield.

\section{Conclusion}

$\mathrm{RhCl}_{3}$, immobilized on an iota-carrageenan xerogel by a very simple and straightforward method, was tested in a Suzuki cross-coupling reaction in various reactor configurations. It was found that increasing the solvent volume from $50 \mathrm{ml}$ to $200 \mathrm{ml}$ while using a simple magnetically stirred system and retaining the reactants and the catalyst amounts, only slightly changed the product yields of the homogenous system. Yet the immobilization of the catalyst decreased the product yield due to mass transfer limitations. Shaking the reaction mixture instead of magnetically stirring it yielded better mixing of the heterogeneous system, but almost the same product yield. The best product yields were observed using the mechanically stirred configuration in a Buchi reactor. Furthermore, the catalysts were easily separated from the reaction mixture and recycled, without catalyst loss, while retaining their catalytic performance characteristics. Finally, use of a tabular reactor, in which the reaction mixture was circulated through the xerogel, also yielded high product yields and allowed easy catalyst recycling. Such a setup can resemble the continuous mode systems which have wide current applications in industry.

One of the main obstacles to implementation of new heterogeneous systems at industrial scales is to prepare relatively simple and cheap catalysts that can work in different high-scale reactors. As most of the new polysaccharide-based heterogeneous catalysts that were offered recently in the literature have been examined only at very small scales, it is important to check them also under other configurations. Thus, the significance of these results is considerable for developing new systems for processes meant to be applied at industrial scales. These findings break new ground and call for further investigation. Adapting natural materials for such "green catalysis" can be a substantial boon for industry and lead to far more sustainable methods of production.

\section{Supporting Information}

Supporting information accompanies this paper on http://www.acgpubs.org/journal/organiccommunications 


\section{ORCID}

Oshrat Levy-Ontman: 0000-0002-3977-7066

Eduard Arbit: 0000-0002-9686-9776

Sivan Leviev: 0000-0001-5977-2134

Adi Wolfson: 0000-0002-8013-0846

\section{References}

[1] Beller, M.; Bolm, C. Transition metals for organic synthesis: Building blocks for fine chemicals, John Wiley \& Sons, 1998.

[2] Hegedus, L. S. Transition metals in the synthesis of complex organic molecules, University Science Books, 1999.

[3] Pierluigi, B.; Liguori, F. Heterogenized Homogeneous catalysts for fine chemicals production: Materials and processes, Springer Science \& Business Media, 2010.

[4] Hübner, S.; de Vries, J. G. Farina, V. Why does industry not use immobilized transition metal complexes as catalysts? Adv. Synth. Catal. 2016, 358, 3-25.

[5] De Vos, D.; Vankelecom, I. F. J.; Jacobs, P. A. Chiral catalyst immobilization and recycling, John Wiley \& Sons, 2008.

[6] Niaounakis, A. K.; Biopolymers: Applications and trends, William Andrew Books, 2015.

[7] Mohanty, M.; Misra, M.; Drzal, L. T. Natural fibers, biopolymers, and biocomposites, Boca Raton, CRC press, 2005.

[8] Lin, N.; Huang, J.; Dufresne, A. Preparation, properties and applications of polysaccharide nanocrystals in advanced functional nanomaterials: A review. Nanoscale 2014, 4, 3274-3294.

[9] Molnár, Á.; Papp, A. The use of polysaccharides and derivatives in palladium-catalyzed coupling reactions. Catal. Sci. Tech. 2014, 4, 295-310.

[10] Miller-Chou, B. A.; Koenig, J. L. A review of polymer dissolution. Progress in Polymer Science 2013, 28, 1223-1270.

[11] Wolfson, A.; Levy-Ontman, O. Recent developments in the immobilization of palladium complexes on renewable polysaccharides for Suzuki-Miyaura cross-coupling of halobenzenes and phenylboronic acids. Catalysts 2020, 10, 136-161.

[12] Wolfson, A.; Levy-Ontman, O. Development and application of palladium nanoparticles on renewable polysaccharides as catalysts for the Suzuki cross-coupling of halobenzenes and phenylboronic acids. Mol. Catal. 2020, 493, 111048-111062.

[13] Rafiee, F. Recent advances in the application of chitosan and chitosan derivatives as bio supported catalyst in the cross coupling reactions. Curr.Org. Chem. 2019, 4, 390-408.

[14] Rafiee, F.; Hosseini, S. A. CNC pincer palladium complex supported on magnetic chitosan as highly efficient and recyclable nanocatalyst in C-C coupling reactions. Appl Organomet. Chem. 2018, 11, 45194529.

[15] Suzuki, A. Organoborane coupling reactions (Suzuki coupling). Proceedings of the Japan Academy, Series 2004, vol. 80, 359-371.

[16] Miyaura, N.; Suzuki, A. Palladium-catalyzed cross-coupling reactions of organoboron compounds. Chem. Rev. 1995, 95, 2457-2483.

[17] Wolfson, A.; Dlugy, C. Palladium-catalyzed Heck and Suzuki coupling in glycerol. Chem. Papers 2007, $61,228-232$.

[18] Gujral, S. S.; Khatri, S.; Riyal, P.; Gahlot, V. Suzuki cross coupling reaction-a review. Indo. Glob. J. of Pharm. Sci. 2012, 2, 351-367.

[19] Lv, J. J.; Wang, V. J.; Feng, J. J.; Qiu, R.; Wang, A. J.,; Xu, X. Facile synthesis of highly active Pd-Cu nanowires catalyst through a simple wet-chemical strategy for ligand-free Suzuki cross coupling reaction. Appl. Catal. A: General 2016, 522, 188-193.

[20] Wang, Z. J.; Wang, X. Y.; Wang, X.; Liang, Z. W.; Xu, X. Carbonylative Suzuki cross-coupling reaction catalyzed by bimetallic Pd-Pt nanodendrites under ambient CO pressure. Catal. Commun. 2017, 101, 1014

[21] Fuerstner, A.; Krause, H. Practical method for the rhodium-catalyzed addition of aryl-and alkenylboronic acids to aldehydes. Adv. Synth.Catal. 2001, 343, 343-350.

[22] Ueura, K.; Satoh, T. Miura, M. Rhodium-catalyzed arylation using arylboron compounds: Efficient coupling with aryl halides and unexpected multiple arylation of benzonitrile. Org. Lett. 2005, 7, 2229-2231, 2005. 
[23] Oh, C. H.; Lim, Y. M.; You, C. H. Platinum-catalyzed cross-couplings of organoboronic acids with aryl iodides. Tetrahedron Lett. 2002, 43, 4645-4647.

[24] Baghbanzadeh, M.; Pilger, C.; Kappe, C. O. Rapid nickel-catalyzed Suzuki-Miyaura cross-couplings of aryl carbamates and sulfamates utilizing microwave heating. The J. Org. Chem. 2011, 76, 1507-1510.

[25] Zultanski, S. L.; Fu, G. C. Nickel-catalyzed carbon-carbon bond-forming reactions of unactivated tertiary alkyl halides: Suzuki arylations. J. Am. Chem. Soc. 2013,135, 624-627.

[26] Thathagar, M. B.; Beckers, J.; Rothenberg, G. Copper-catalyzed Suzuki cross-coupling using mixed nanocluster catalysts. J. Am. Chem. Soc 2002, 124, 11858-11859.

[27] Levy-Ontman, O.; Biton, S.; Shlomov, B.; Wolfson, A. Renewable polysaccharides as supports for palladium phosphine catalysts. Polymers 2018, 10, 659-674.

[28] Wolfson, A.; Biton, S.; Levy-Ontman, O. Study of Pd-based catalysts within red algae-derived polysaccharide supports in a Suzuki cross-coupling reaction. RSC Advances 2019, 8, 37939-37948.

[29] Wolfson, A.; Pierschel, E,; Orzehovsky, T.; Nirenberg, S. Levy-Ontman, O. Heterogeneous iota carrageenan-based palladium catalysts for organic synthesis. Org. Commun. 2019, 12, 149-159.

[30] Leviev, S.; Wolfson, A. Levy-Ontman, O. RhCl(TPPTS) 3 supported on iota-Carrageenan as recyclable catalysts for Suzuki cross coupling. J.Appl. Polymer Sci. 2019, 136, 48200-48204.

[31] Leviev, S.; Wolfson, A.; Levy-Ontman, O. Novel iota carrageenan-based $\mathrm{RhCl}_{3}$ as an efficient and recyclable catalyst in Suzuki cross coupling. Mol. Catal. 2020, 486, 110841-110849.

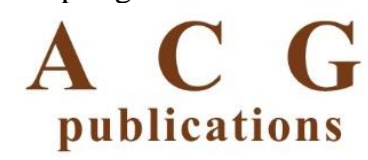

(C) 2021 ACG Publications 